\title{
Fog Detection Using Airport Radar
}

\author{
G. Galati", I. Dalmasso", G. Pavan", G. Brogi** \\ * "Tor Vergata" University of Rome \\ Via del Politecnico, 1 - 00133 Roma, ITALY \\ e-mail: galati@disp.uniroma2.it \\ ** SELEX Sistemi Integrati S.p.A. \\ Via Tiburtina Km.12.400 - 00131 Roma, ITALY \\ e-mail: gbrogi@selex-si.com
}

\begin{abstract}
Fog is a significant factor affecting the Air Traffic Control (ATC). Significant limitations of the airport capacity are due to fog that causes the reduction of the visibility (Runway Visual Range, RVR). Today METAR (Meteorological Aviation Report) and forecasts TAF (Terminal Aerodrome Forecast) are only available to estimate the visibility variations, but not to estimate the RVR. Scope of this paper is to evaluate the performance of airport radar sensors to detect the fog.
\end{abstract}

\section{Introduction}

Significant limitations of the airport capacity are due to fog, i.e. small water particles in the air, specially when close to the land surface. The presence of these particles causes the scattering of the light and therefore the reduction of the visibility (that is a significant reduction for Airport/ATC operations when less than $1 \mathrm{~km}$ ).

The formation of a fog layer happens when a humid air mass comes cooled until saturation, that is at the dew temperature. This cooling can be due to both a radiation or an advection process of warm air masses over cold surfaces. In literature the fog is classified in four types: advection fog (strong and light) and radiation fog (strong and light) [1].

On the airport surface the presence of the fog reduces the Runway Visual Range (RVR), affecting the airport flow (a single movement at any time is allowed in low visibility, thus strongly reducing the capacity). Today METAR (Meteorological Aviation Report) and forecasts TAF (Terminal Aerodrome Forecast) are only available to estimate the visibility variations, but not to estimate the $R V R$.

Scope of this paper is to evaluate the performance of the airport radar sensors to detect the fog. In particular we are consider the following systems: two radars for the control of airport surface (SMR-Surface Movement Radar) at $X(9 \mathrm{GHz})$ and $\mathrm{W}(95 \mathrm{GHz})$ band.

\section{Fog models and radar observables}

In order to represent the different types of fog, the models studied from Silverman and Sprague [1] use modified Gamma distribution to describe the size distribution of the particles (Drop Size Distribution, DSD), i.e.: $N(r)=A \cdot r^{\alpha} \exp \left(-b \cdot r^{\gamma}\right)$, where $r$ is the radius $(\mu m)$, $\alpha$, $b$ and $\gamma$ are the positive parameters while $A$ is a normalization constant $\left(\mathrm{cm}^{-3} \mu m^{-1-\alpha}\right)$. The $D S D$ parameters for the four types of fog are shown in Table 1 . In the following, we will use the diameter $D$ instead of radius $r$. Being the diameters of the particles $D(<100 \mu \mathrm{m})<<\lambda(3 \mathrm{~cm}$ at $X$-band and $3 \mathrm{~mm}$ at $W$-band) the radar cross section $(R C S)$ of the $i^{\text {th }}$ particle is given by Rayleigh approximation: $\sigma_{i}=\frac{\pi^{5} D_{i}^{6}}{\lambda^{4}}|K|^{2}$ with $|K|^{2}$ related to the complex refraction index 
that depends on temperature. When $N(D)$ is known, the reflectivity per unit volume $\eta$, results defined by: $\eta=\frac{\pi^{5}|K|^{2}}{\lambda^{4}} \int D^{6} N(D) d D$ where the integral term (the $6^{\text {th }}$ moment of the $D S D$ ) represents the reflectivity factor $Z$ (that does not depend on frequency); normally $Z$ is measured in $\mathrm{mm}^{6} \mathrm{~m}^{-3}$ or in $d B z$ as $Z_{d B z}=10 \log _{10}\left(Z_{m m^{6} m^{-3}}\right)$. The $3^{r d}$ moment of $N(D)$ is related to the liquid water content: $L W C=\frac{2}{3} \rho_{w} \pi \int D^{3} N(D) d D$ where $\rho_{w}$ is the water density. Considering the four models of the fog, the values of the reflectivity factor $Z$ (in $d B Z$ ) and the $L W C$ (in $\mathrm{g} \mathrm{m}^{-3}$ ) are shown in Table 1.

\begin{tabular}{|l|l|c|c|c|c|c|c|c|}
\hline Type offog & Model & $\boldsymbol{A}$ & $\boldsymbol{\alpha}$ & $\boldsymbol{b}$ & $\boldsymbol{\gamma}$ & $\boldsymbol{Z}(\boldsymbol{d B z})$ & $\boldsymbol{L} \boldsymbol{W C}\left(\mathbf{g ~ m}^{-3}\right)$ & $\boldsymbol{V}(\mathbf{k m})$ \\
\hline \multirow{2}{*}{ ADVECTION } & 1: strong & 0.06592 & 3 & 0.3 & 1 & -3.03 & 0.91 & 0.02 \\
\cline { 2 - 9 } & 2: light & 0.027 & 3 & 0.375 & 1 & -16.79 & 0.078 & 0.089 \\
\hline \multirow{2}{*}{ RADIATION } & $\begin{array}{l}\text { 3: strong } \\
\text { 4: light }\end{array}$ & 2.37305 & 6 & 1.5 & 1 & -31.27 & 0.062 & 0.146 \\
\cline { 3 - 9 } & 407.5 & 6 & 3.0 & 1 & -46.32 & 0.016 & 0.352 \\
\hline
\end{tabular}

Table 1 - DSD Gamma parameters, Reflectivity Z, LWC and visibility V for the considered four models of the fog.

\section{Visibility and Attenuation due to fog}

An object is visible when the contrast with the surrounding environment is still sufficiently high. So the visibility $V$ is defined as the distance to which the contrast ratio between a black object and a clear background is equal to 0.02 . Empirical relations between visibility $V(\mathrm{~km})$ and liquid water content $L W C\left(\mathrm{gm}^{-3}\right)$ have been evaluated by Currie [2]. Using these relations, i.e.: $V=0.017 \cdot L W C^{-0.65}$ for advection fog and $V=0.024 \cdot L W C^{-0.65}$ for radiation fog, in the last column of Table 1 the values of the visibility are reported.

The attenuation phenomena depend on frequency. At $X$-band the attenuation due to fog is neglected. For example the values of the specific attenuation for strong advection fog are $0.112 \mathrm{~dB} / \mathrm{km}, 0.09 \mathrm{~dB} / \mathrm{km}, 0.08 \mathrm{~dB} / \mathrm{km}$ and $0.07 \mathrm{~dB} / \mathrm{km}$ when the temperature is $-10{ }^{\circ} \mathrm{C}, 0{ }^{\circ} \mathrm{C}$, $5^{\circ} \mathrm{C}$ and $10^{\circ} \mathrm{C}$ respectively. While at $95 \mathrm{GHz}$ the specific attenuation results of $4.84 \mathrm{~dB} / \mathrm{km}$, $4.35 \mathrm{~dB} / \mathrm{km}, 4.08 \mathrm{~dB} / \mathrm{km}$ and $3.83 \mathrm{~dB} / \mathrm{km}$ considering the same temperatures and fog models [3]. These values has computed by: $A_{f o g}=K_{l} \cdot L W C(\mathrm{~dB} / \mathrm{km})$ with $L W C$ in $\left(\mathrm{gm}^{-3}\right)$ where $K_{l}=6.0826 \cdot 10^{-4} \cdot f^{1.8963} \cdot \gamma^{\left(7.8087-0.0156 f-3.0731^{-4} f^{2}\right)}$ is expressed in $d B / \mathrm{km} / \mathrm{g} / \mathrm{m}^{3}$ with $\gamma=300 / T, T$ in Kelvin and $f$ in $\mathrm{GHz}$ [3].

\section{Detection of fog}

Radar equation allows to evaluate the received power due to the scattering of fog particles. Supposing a Gaussian antenna pattern the power received is [4]:

$$
P_{r}(m W)=3.99 \cdot 10^{-20} \frac{P_{t}(W) G^{2}(\operatorname{lin}) \tau(\mu s) \theta(\mathrm{deg}) \phi(\mathrm{deg})}{\lambda^{2}(\mathrm{~cm})} L^{2}|K|^{2} \frac{Z\left(\mathrm{~mm}^{6} \mathrm{~m}^{-3}\right)}{R^{2}(\mathrm{~km})}
$$

where $P_{t}$ is the transmitted power, $G$ is the gain, $\theta, \phi$ are $-3 d B$ beamwidth, $\tau$ is the pulse width and $\lambda$ the wavelength; $L$ is the coefficient of attenuation. The maximum range $\left(R_{\max }\right)$ 
has been evaluated for a Signal to Noise Ratio $(S N R)$ of $10 \mathrm{~dB}$ and for both constant and variable fog distribution along the range. The considered radars are installed in various Italian airports (e.g. Fiumicino and Malpensa for $X$-band, Venice and Linate for the $W$-band).

\section{Case A) Constant fog profile along the path}

$\operatorname{SMR} X$-band $\left(\lambda=3.3 \mathrm{~cm} ; G=34 \mathrm{~dB} ; P_{t}=20 \mathrm{~kW} ; \tau=40 \mathrm{~ns} ; \theta=0.4^{\circ} ; \phi=22^{\circ}\right)$ has shown a reduced maximum range: $R_{\max }=500 \mathrm{~m}$ in the more intense (strong advection) fog case, $R_{\max }=100 \mathrm{~m}$ for weak advection fog, $R_{\max }<50 \mathrm{~m}$ for radiation fog (strong and weak), using linear polarization in transmission. In case of circular polarization in transmission and copolar polarization (i.e. rain suppression configuration) in reception the values of $R_{\max }$ are lower. To enhance fog detection performance, this $X$-Band SMR radar would need for a cross polar channel that is difficult to implement in the standard, 6 meters long (for the classical $0.4^{\circ}$ beam width) slotted wave guide antennas. On the other hand, reflector antennas for $X$ band SMR are very seldom used due to their high cost.

The use of the millimetre waves ( $W$-band) in surface radar permits a small reflector antenna (reflector width $1 \mathrm{~m}$ for a $0.22^{\circ}$ beamwidth) and ease of installation. With a circular polarization transmission, an orthomode junction in the antenna feed permits to feed two receiving channels, a copolar for the detection of targets (aircraft, vehicles) and minimum sensitivity to rain, and a crosspolar for maximum sensitivity to the fog echo. The maximum range for this configuration is: $R_{\max }=2.5 \mathrm{Km}$ in the strong advection fog case, $R_{\max }=500 \mathrm{~m}$ for weak advection, $R_{\max }<100 \mathrm{~m}$ for radiation fog (strong and weak). However $W$-band $S M R$ suffers from attenuation effects and ground clutter.

\section{Case B) Variable fog profile along the path}

A temperature of $5{ }^{\circ} \mathrm{C}$ and a range profile of $6 \mathrm{~km}$ within 500 range cells of $12 \mathrm{~m}$ have been supposed. Along the range $D S D$ parameters $A$ and $b$ in our analysis have been linearly varied from cell to cell so that the $D S D$ changes from model 2 to model 1 (see $D S D$ plots in Figure 1). Subsequently the $3^{r d}$ and the $6^{\text {th }}$ moment of the $D S D$ are estimated to calculate the $L W C$ and the reflectivity $Z$. By means of $L W C$ the visibility are evaluated by equation $V=0.017 \cdot L W C^{-0.65}$. Figure 2 shows the visibility profile along the path (expressed in number of range cells, 1 range cell $=12 \mathrm{~m}$ ).

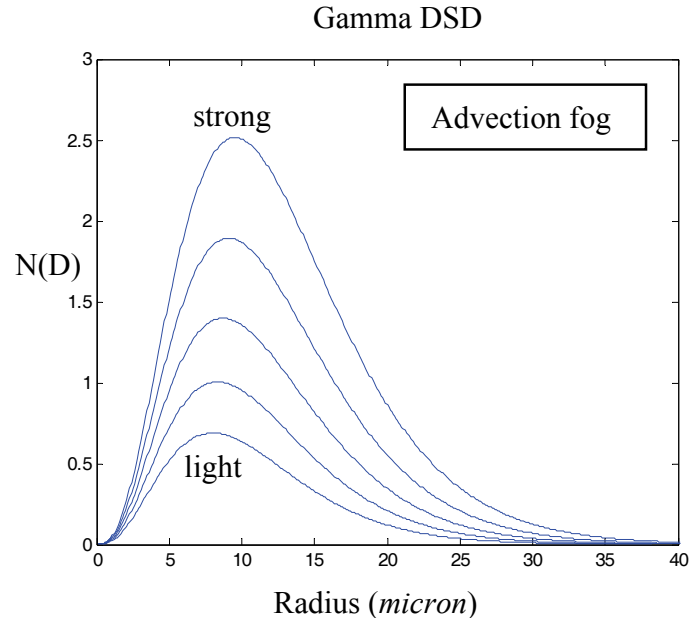

Figure 1 - DSD of fog.

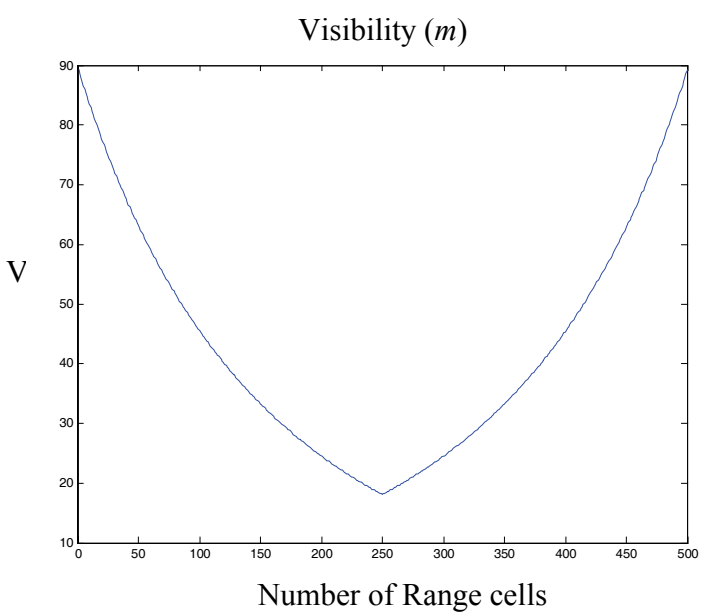

Figure 2 - Visibility range profile. 
Considering at $W$-band the cumulative attenuation along the path and employing equation (1), Figure 3 compares the power received with and without attenuation versus the number of range bins.

In Figure 4 the Signal to Noise Ratio $(S N R)$ versus range $(\mathrm{km})$ is shown. The maximum range detected evaluated for a $S N R$ of $10 \mathrm{~dB}$, results around $3 \mathrm{~km}$.

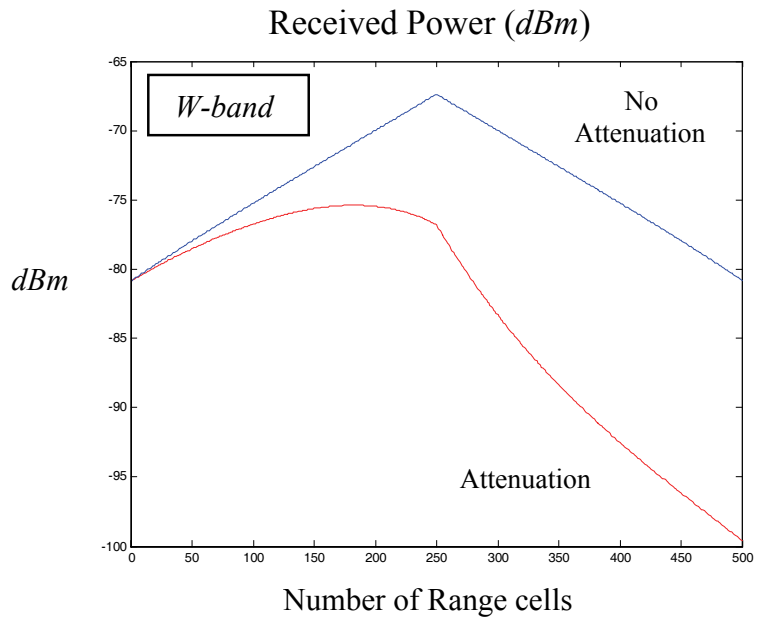

Figure 3 - Received power ( $\mathrm{dBm}$ ) vs N. of Range cells.

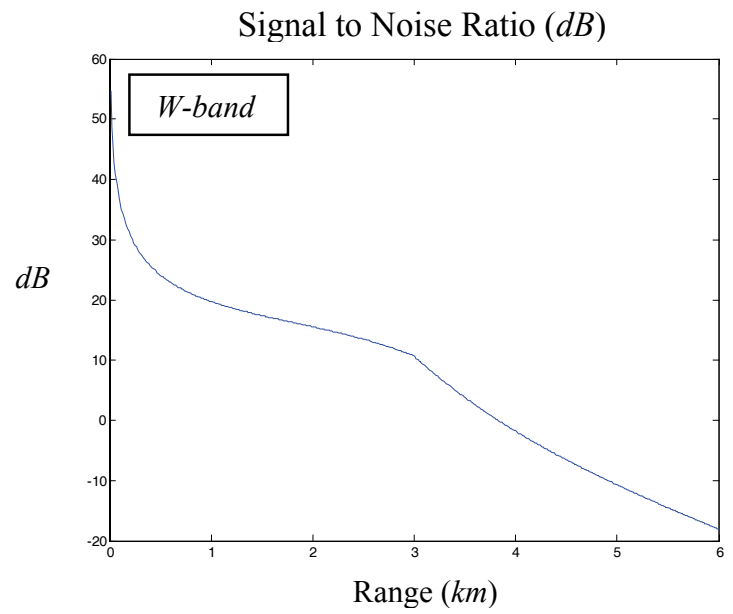

Figure 4 - SNR $(d B)$ vs Range $(\mathrm{km})$.

\section{Conclusions}

A Surface Movement Radar can be adapted to detect fog banks and to track their evolution, to help management of airport operations. However, effective detection is only possible by using:

a) cross-polar circular polarization in reception (therefore two channel are needed: for target and for fog);

b) millimeter waves (i.e. at $\mathrm{W}$-band around 94 - $95 \mathrm{GHz}$ or even higher);

c) a network of small sensor to cover the whole airport area and its surrounds.

\section{Reference}

[1] Shettle P., Fenn W. "Models for the aerosols of the lower atmosphere and the effects of the humidity variations on their optical properties", Optical Physics Division, Project 7670, Air Force Geophysics Laboratory, 1979.

[2] Currie C., Brown E. "Principles and Applications of Millimeter-Wave Radar", Artech House, 1987.

[3] Zhao Z., Wu Z. "Millimeter-wave attenuation due to fog and clouds", International Journal of Infrared and Millimeter Waves, Vol. 21, No. 10, 2000.

[4] Sauvageot H. "Radar Meteorology", Artech House, 1992. 